clusions as to the nature of the meningeal infection from these findings. This does not, however, materially influence the point of importance which the author desired to bring out, namely, that it is wise in secondary meningitis to use intraspinally the autogenous serum, which probably contains a number of antibodies from the primary infection.

Conclusion. We recommend the use of autogenous serum injected into the subarachnoid space in cases of secondary meningitis when there is no efficient specific immune serum.

I must take this opportunity to thank Capt. A. L. Garbat, chief of the laboratory of U. S. A. General Hospital No. 12 for his ever-helpful coöperation. I also appreciate the assistance given by Sgt. P. Piel and his brother Pvt: R. Piel, of the laboratory force, who carefully made up the autogenous serum under our direction.

\title{
OBSERVATIONS ON THE COLLOIDAL GOLD REACTION WITH CEREBROSPINAL FLUID.
}

\author{
By Ellis Kellert, M.D., \\ ALBANY, NEW YORK.
}

(From the Bender Hygienic Laboratory, Albany, N. Y.)

DURING the past four years the colloidal gold test on cerebrospinal fluid as devised by Carl Lange has aroused great interest among physicians and laboratory workers. This reaction, the exact nature of which is not yet clearly understood, is proving of some practical value as a diagnostic procedure. The utility of the test is not generally recognized at the present time, and frequently the reaction yields information not obtained so quickly by any other diagnostic measure. This is seen particularly in the occasional case of meningitis in which the Wassermann reaction is negative and a characteristic curve is obtained by the colloidal gold method, or where a tuberculous curve is obtained and no acid-fast bacilli found; also in cases in which differential diagnosis between poliomyelitis and tuberculous meningitis is difficult the reaction has proved of value. As in all laboratory diagnostic procedures, however, occasional false reactions are obtained, and it is the chief object of this paper to record a few such instances and to make certain observations which may prove of interest to those performing the test.

Previous articles on the gold reaction have considered in great detail the methods of preparing the fluid, and we shall not discuss the subject except to say that if directions regarding cleanliness of glassware and purity of reagents are carefully adhered to no great 
difficulty will be experienced in making the colloidal gold. Colloidal fluids will vary in their physical characteristics, and occasionally the same spinal fluid will give, with different colloidal gold solutions, a slightly variable curve, but the results are usually comparable. Nevertheless, it is advisable to use spinal fluid from known cases as a control to check the accuracy of each new lot of colloidal gold. Fluids that are opalescent yield a more marked curve than others, but although exaggerated the curve can usually be interpreted. Conversely, fluids having a clear red appearance and no trace of opalescence may yield but slight and inconclusive reactions. Such fluids are much more permanent than those possessing some degree of opalescence. In preparing the fluid it will be found that quantities of 500 c.c. or less are more readily prepared than larger amounts, and because of the ease of manipulation, this quantity is recommended or smaller quantities when few specimens are received.

Negative Tests. In our experience the curves obtained in tuberculous and syphilitic meningitis and in tabes have been similar to those described by others. No correlation could be made between the degree of the curve and the extent of pathological involvement, but the fluid from patients with tuberculous meningitis gave an exceedingly pronounced curve when examined several days before death. In fact the reactions might be termed atypical, in that they tended to show the greatest change in the terminal tubes. Such fluids also showed a greatly increased cell count and positive globulin reaction, and the extent of the curve seemed to run parallel with the amount of globulin present cstimated by the Noguchi butyric acid method. In a series of seventy-five fluids giving ncgative colloidal gold reactions only three showed a positive globulin test and increased cell count. They were as follows: one of syphilis, one of poliomyelitis and one of tuberculous meningitis. Thus the reaction failcd in 4 per cent. of fluids that were obtained from known cases positive by other methods. In other instances of negative curve and positive globulin test, blood in greater or lesser quantity was found on microscopic examination. In the examination of the centrifugalized sediment it is highly important, in determining the presence of blood, to examine a fresh, unstained drop for very often in routine work the presence of a moderate number of erythrocytes is not demonstrated by the staining methods ordinarily used. 'The erythrocytes do not stain well or are washed off during the various manipulations. In our seventy-five negative fluids, which included a variety of conditions, there were three which gave positive Wassermann reactions and two others giving negative Wassermann tests, though the blood in both these cases yielded positive Wassermann reactions. These latter fluids reacted negatively by all other methods. The colloidal gold test, therefore, failed to react in 7 per cent. of positive cases. 
SyphiLis. Fairly constant reactions were obtained in syphilis, but there was great variation in the extent of the curves, and here, again, it appeared that the most marked reactions gave the strongest globulin tests. In a total of 122 luetic curves of varying degree there were obtained 95 giving changes that could be regarded as positive or highly suggestive of syphilis. Of these 62 , or 70 per cent., gave positive Wassermann reactions, and of the remainder, 24 per cent., could be excluded as probably not syphilitic. There thus remained 6 per cent. of specimens when the colloidal gold test was positive and the Wassermann reaction negative. These cases were syphilis clinically. In 22 per cent. of the fluids the curves could not be interpreted, being either slight or else irregularly exaggerated. These figures would indicate that while the method appears to be more sensitive than the Wassermann test, there is much greater possibility of error in the absence of confirmation by other methods.

Tuberculosis. Forty cerebrospinal fluids were received which gave positive colloidal gold curves and in which the tentative clinical diagnosis of tuberculous meningitis was made. Of these, 7 were subsequently excluded as not tuberculous. There thus remained 33 cases of tuberculous meningitis corroborated by othcr" tests, guinea-pig inoculations or postmortem examinations. All these fluids yielded a negative Wassermann reaction, but had an increased cell content and globulin. Of the 33 known cases, 4 gave curves suggesting acute meningitis. Thus the reaction was correct in 88 per cent., and misleading in 12 per cent. The four fluids yielding acute curves were from terminal cases, and all showed a greatly increased cell count, strongly positive butyric acid test and tubercle bacilli in smears. Guinea-pigs were also positive for tuberculosis.

Acute Meningitis Fluids from 27 cases of acute meningitis were examined and 20 , or 74 per cent., gave curves suggesting acute meningitis. Thus 26 per cent. were negative or inconclusive. In all these specimens the organisms were demonstrated in smears or cultures except one. In addition to the meningococcus there was 1 case of pneumococcus meningitis and 1 of Bacillus mucosus capsulatus infection. The latter reacted negatively. One fluid gave a curve in the luetic zone and 3 others were difficult to distinguish from tuberculous curves. The one fluid in which no organisms were found and which did not yield a characteristic curve showed a strongly positive globulin test, heavy sediment of polynuclear leukocytes and no reduction of Fehling's solution. This patient, a young woman of sixteen years, had been ill two weeks and subsequently became well without serum treatment.

Thus we find the reaction confirmatory in approximately 80 per cent. of cases. It apparently is most valuable in cerebrospinal syphilis, for many positive results are obtained when the complement-fixation reaction is negative. These results, however, unless confirmed by positive globulin tests and increased cell count, 
should not form the basis for intraspinal or intravenous medication, but should lead to very careful observation of the patient and repeated examination of the fluid. In tuberculous meningitis the chief value of the reaction lies in the fact that a majority of these cases may quickly be distinguished from poliomyelitis in children and syphilis in adults. It thus also becomes valuable from a prognostic standpoint, but guinea-pig inoculation should not be neglected even in such instances. In acute meningitis the other changes are of much greater importance. It is rather interesting to note that in tuberculous meningitis when the exudate becomes very profuse the gold curve suggests acute meningitis. Differential diagnosis at this time would be difficult without the presence of organisms in smears. In poliomyelitis we found the curve inconstant, but more often in the luetic zone. In a number of instances in which the condition could not be differentiated clinically the curve was misleading. A few of the more interesting of these cases are cited below.

CASE I.-Mr. H. J., aged forty-two years. Chief symptoms: mental confusion; marked headache; retraction of head; bilateral optic neuritis; slight nystagmus, chiefly to left; reflexes weakened; evening temperature. 'The clinical diagnosis was meningitis. Four specimens of spinal fluid were received over a period of twenty three days, with the following results:

\begin{tabular}{c|c|c|c|c|c|c}
\hline & Globulin. & Cell count. & \multicolumn{2}{|c|}{ Smears. } & Wasser mann & Colloidal gold. \\
\hline 1 & Positive & $\ldots$ & $\begin{array}{c}\text { Increased number of cells, fow } \\
\text { mononuclears and many poly- } \\
\text { nuclears, few erythrocytes }\end{array}$ & Negative & 0035554300 \\
& & & do. & do. & Negative & 1543333210 \\
2 & Positive & 460 & do. & Negative & 0034543100 \\
3 & Positive & 360 & do. & do. & Negative & 0014544333 \\
\hline
\end{tabular}

No organisms were found in smears, cultures were sterile and guinea-pigs inoculated showed no evidence of tuberculosis. The patient died and the autopsy disclosed a subacute endocarditis involving the mitral valve and numerous infarctions of the brain. In this case the curves obtained were entirely misleading. At different times tuberculosis, syphilis and acute meningitis were suggested.

CASE II.-George K., aged two years. Clinical diagnosis: acute meningitis. The patient presented the usual symptoms of acute meningitis. The first spinal fluid received was very turbid; globulin strongly positive; cell count, 140 . Wassermann negative. The sediment showed numerous small and large mononuclear cells, polynuclears and no organisms. The following gold curve was obtained: 0123344554. A similar result was obtained seventeen days later, but acid-fast bacilli were also found. The patient died and the autopsy disclosed tuberculous meningitis, with a very extensive exudate at the base of the brain. This, therefore, was a 
case of tuberculous meningitis, but the colloidal gold curve suggested acute meningitis.

CASE III.-Miss B., aged seventeen years. Eight weeks after operation for middle-ear abscess the patient developed intense headache; increased reflexes; tempcrature $100^{\circ}$ to $100.5^{\circ} \mathrm{F}$; photophobia. Five specimens of spinal fluid and one of blood examined over a period of one month gave negative Wassermann reactions. The globulin reactin was strongly positive and the sediments showed greatly increased numbers of lymphocytes. The colloidal gold reaction persistently suggested syphilis, giving curves of which the following is an example, 5544311100. Six guinea-pigs inoculated with different specimens of spinal fluid gave negative results. The patient died shortly after, but no autopsy could be obtained. The history and termination suggested cerebral abscess, but the colloidal gold test suggested tabes.

CASE IV.-Mrs. C. P., aged forty-four years. Clinical diagnosis not made. Syphilis suspected. Chief symptoms: headache; vomiting; inability to walk; exaggerated reflexes; weakness. Temperature, $97^{\circ} \mathrm{F}$. "The Wassermann reaction was reported negative by two different laboratories." The cell count and globulin were increased and the colloidal gold reaction gave the following curve2345542000 - thus suggesting syphilis. About six weeks later the patient died. The history and physical signs in this case suggested cerebral tumor. No autopsy could be obtained.

CASE V. The following data are of interest as an illustration of the effect of intraspinous medication. Mr. G., aged forty-five years. Primary lesion seventeen years previously. Symptoms at present suggest taboparesis.

\begin{tabular}{|c|c|c|c|c|c|c|c|c|}
\hline \multicolumn{5}{|c|}{ Date. } & Globulin. & Wassermann. & Cell count. & Colloidal gold. \\
\hline June & 9 & . & . & . & Positive & ++++ & 250 & 4555543100 \\
\hline & 22 & . & . & - & Positive & $++t+$ & Greatly increased & 2345554310 \\
\hline July & 12 & . & & . & Positive & +++ & 25 & 0001222210 \\
\hline & 20 & . & & . & Positive & ++ & Slight increase & 3333200000 \\
\hline Aug. & 3 & . & & . & Negative & $\ldots$ & 80 & 0000000000 \\
\hline Sept. & 1 & . & . & . & $\begin{array}{l}\text { Weakly } \\
\text { positive }\end{array}$ & ++++ & $\ldots$ & 3333300000 \\
\hline & 21 & . & • & . & Positive & $+t+t$ & Increased & 1133331110 \\
\hline Nov. & 17 & • & & & Positive & $+t+$ & & \\
\hline Dec. & 19 & . & 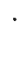 & • & $\begin{array}{l}\text { Weakly } \\
\text { positive }\end{array}$ & ++ & Increased & 1333331000 \\
\hline
\end{tabular}

This patient received numerous intraspinous injections of salvarsanized serum and intramuscular injections of mercury salicylate. The treatment appeared to have but slight permanent action on the syphilitic process. It is interesting to note that the extent of the colloidal gold curves remained in keeping with the Wassermann reaction and the globulin test. 
Case VI.-Mr. T. H., aged forty years. Died, after several days' illness, with symptoms suggesting meningitis. At autopsy there was found acute pancreatitis and-intense edema of the brain. The cerebrospinal fluid removed at autopsy gave the following curve - 1134555552 - thus suggesting acute meningitis. Otherwise the fluid was negative.

CASE VII.-T. N., aged three years. Symptoms of acute meningitis. The cerebrospinal fluid showed 1100 cells per cubic centimeter, strongly positive globulin test and negative Wassermann reaction. The sediment yielded greatly increased numbers of lymphocytes and polynuclear leukocytes; acid-fast bacilli present. The colloidal gold reaction performed, with two specimens of fluid collected on different days gave negative results. Guinea-pigs were positive for tuberculosis.

CASE VIII.--Miss C. E., aged twenty years. After preliminary malaise, anorexia, headache and mental dulness the patient rapidly developed symptoms of acute meningeal disturbance, with marked retraction of head, rigidity of spine, hyperflexion of legs and thighs in lateral decubitus and progressively increasing stupor. The pupils were immobile, but there was no definite strabismus. Clinical diagnosis: tuberculous meningitis.

The spinal fluid showed greatly increased number of lymphocytes, positive globulin reaction (double plus) and negative Wassermann. The colloidal gold curve suggested syphilis. Guinea-pigs reacted positively for tuberculosis.

CASE IX.-Miss N., aged twelve years. Complete paraplegia below the fourth cervical segment; slow onset; three months' duration. No sensory disturbances. Electrical reactions present. Cerebrospinal fluid under pressure, clear; cell count, ten; globulin strongly positive. Wassermann reaction negative. Colloidal gold curve 1223331100, suggesting syphilis. Clinical diagnosis: poliomyelitis.

CASE X.-Mr. E. C., aged thirty years. Influenza two weeks previously. Entered the hospital complaining of headache, vertigo and vomiting. Temperature, $97^{\circ} \mathrm{F}$; pulse, 50. Two days later he became restless and delirious. Axillary temperature, $100^{\circ} \mathrm{F}$.; pulse, 120; slight retraction of head; twitching of arms and legs, especially on the left side. Respiration rapid and of Cheyne-Stokes type.

The cerebrospinal fluid removed just prior to the onset of the latter symptoms contained eight cells per cubic centimeter; globulin double plus; no reduction of Fehling's solution and a negative Wassermann reaction. Smears showed mononuclears chiefly. No organisms were found. The colloidal gold reaction gave the following curve -0133322100 . The patient died and the autopsy disclosed a diffuse glioma involving the right parietal region. 
Blood Contamination. Perhaps one of the most serious faults in the test lies in the fact that cerebrospinal fluids when contaminated with blood yield curves in the luetic zone, thus making interpretation very difficult. A relatively small quantity of blood serum appears to be sufficient to produce marked reactions. To determine precisely this effect, human serum in varying dilutions was tested with colloidal gold and found to yield curves in the luetic zone. The curves were most typical in a 1 to 1000 dilution, and this quantity of serum is sufficient to give a plus minus or a one plus globulin reaction. In routine work a large proportion of spinal fluids contain a trace of blood, and if not carefully guarded against, serious errors of interpretation may result. Human serum in very low dilution gives curves in the terminal or acute meningitic zone. Diluted luetic serum appears to give more pronounced curves than the non-luetic serum and inactivation does not greatly alter the curves. The following is an example of the gold solution reactions obtained with human serum:

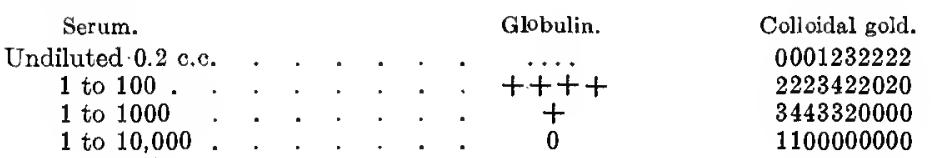

Although it is preferable to perform the reaction with fresh fluid, yet it was found that specimens kept several weeks at ice-box temperature yielded a characteristic but slightly diminished curve. The heating of fluids caused slight change in the curves even when the spinal fluid was heated to the boiling-point. The few observations made with contaminated fluids show that such specimens are unsuited for examination, for frequently reactions are obtained in the luetic zone when all other tests are negative.

Conclusions. 1. The colloidal gold reaction is useful Ias an additional or confirmatory test.

2. It is of greatest value in the syphilitic diseases of the central nervous system, especially tabes and paresis.

3 . The test may serve to differentiate between tuberculous and other forms of meningitis.

4. 'The reaction is correct in approximately 80 per cent. of cases.

5. Cerebrospinal fluid contaminated with blood in small quantity frequently gives reactions in the luetic zone.

6. Positive results unconfirmed by other tests are of only slight value.

7. The Wassermann reaction and the cytological examination of the cerebrospinal fluid are of greater value than the colloidal gold. test. 